\title{
Techniques for Conducting Effective Concept Design and Design-to-Cost Trade Studies
}

\author{
David A. Di Pietro \\ NASA Goddard Space Flight Center, Mail Code 599, Greenbelt MD 20771 \\ Phone: 301 286-0240, E-mail: david.a.dipietro@nasa.gov, ddipietro@erols.com \\ Copyright $\odot 2015$ by David A. Di Pietro. Published and used by INCOSE with permission.
}

\begin{abstract}
Concept design plays a central role in project success as its product effectively locks the majority of system life cycle cost. Such extraordinary leverage presents a business case for conducting concept design in a credible fashion, particularly for first-of-a-kind systems that advance the state of the art and that have high design uncertainty. A key challenge, however, is to know when credible design convergence has been achieved in such systems. Using a space system example, this paper characterizes the level of convergence needed for concept design in the context of technical and programmatic resource margins available in preliminary design and highlights the importance of design and cost evaluation learning curves in determining credible convergence. It also provides techniques for selecting trade study cases that promote objective concept evaluation, help reveal unknowns, and expedite convergence within the trade space and conveys general practices for conducting effective concept design-to-cost studies.
\end{abstract}

\section{Preface}

Concept design may be conducted using a variety of methods, some more efficient and effective than others. Using a space observatory example, this paper describes some of the more strategic aspects of one process for conducting comprehensive concept design and design-to-cost trade studies. This process is best suited for studying immature (e.g., first-of-a kind) mission concepts that advance the state of the art and that have high design uncertainty. Aspects discussed include: a) what concept design is and why it is important, b) the fidelity needed in the concept design solution, c) techniques for designing the mission level trade space, and d) challenges in determining credible design convergence.

\section{What Concept Design is and Why it is Important}

Concept design typically is conducted to determine a "feasible" system level design baseline for a new concept, as described in par. 5.3 of ref. (a). An exploratory process, it is as much about investigating requirements as it is about investigating design. More specifically for space missions, it involves a concurrent investigation of multiple mission characteristics such as design, performance, concept of operations (CONOPS), flight dynamics, technology development, verification approach, launch and ground station interfaces, mission and science operations centers, cost, schedule, and risk. 
Figure 1 shows Pre-Phase A and Phase A of the NASA project life cycle are used to develop the concept design. Phase B is used to develop the preliminary design, and Phase C, up to Critical Design Review (CDR), is used to develop the detailed (or final) design. The post-CDR portion of Phase $\mathrm{C}$ is used to fabricate system elements and prepare for their physical integration.

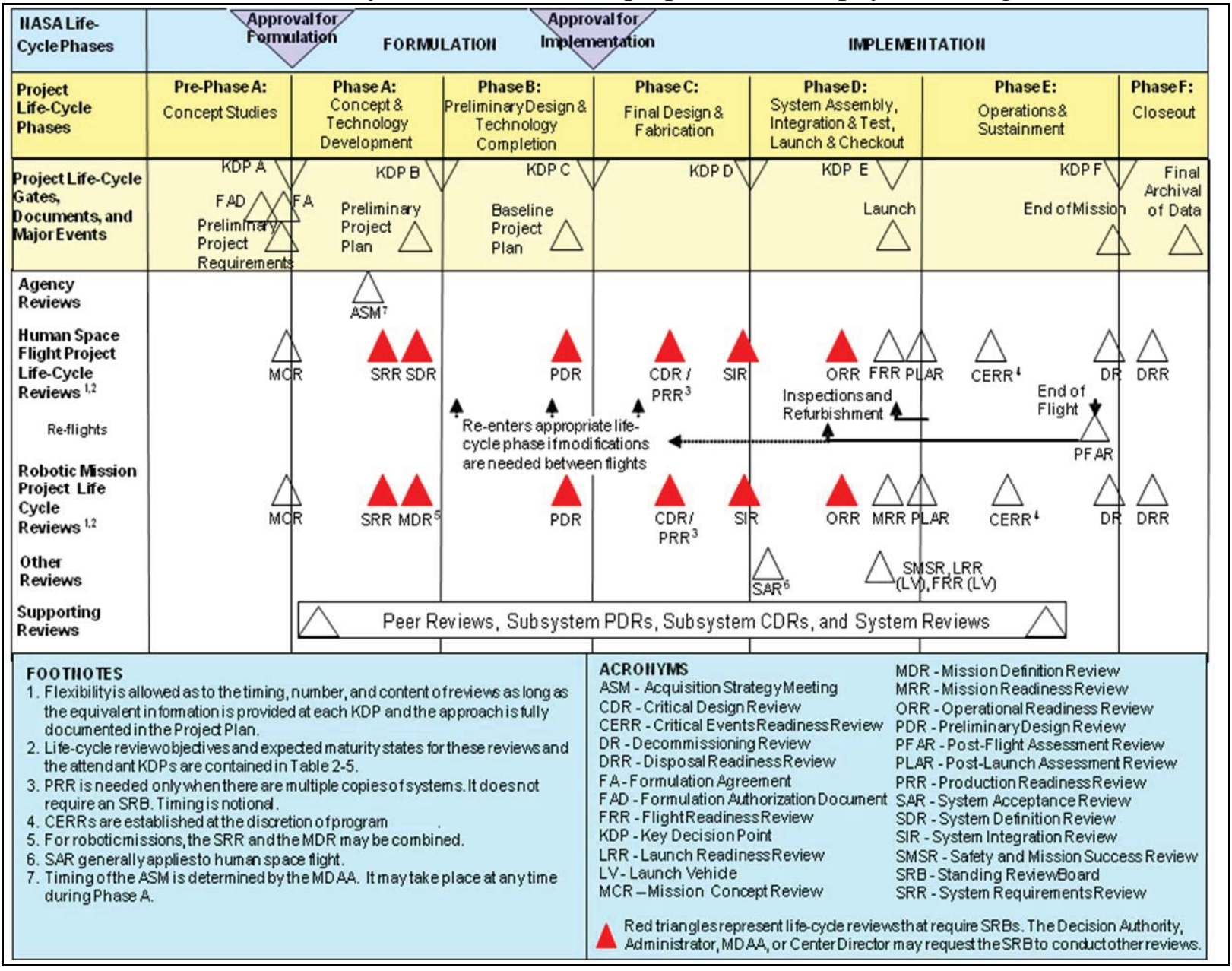

Figure 1: The NASA Project Life Cycle (ref. (b))

Concept Design Determines Majority of Life Cycle Costs. The earliest life cycle phases have the most leverage over life cycle cost. This is conceptually illustrated in Fig. 2, which shows by

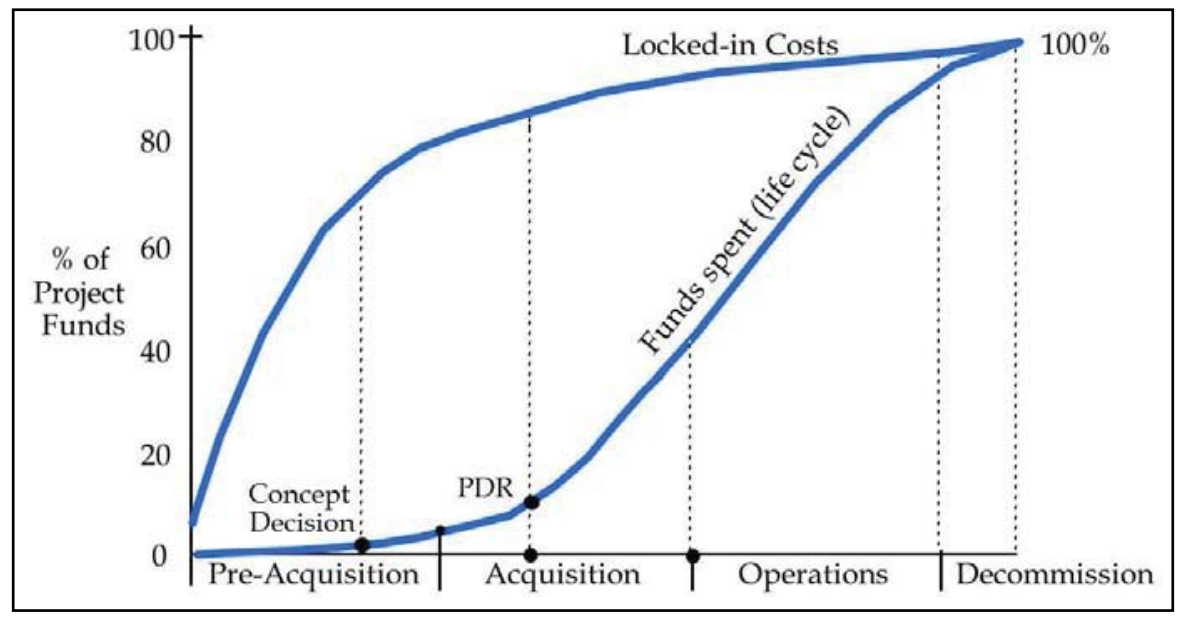


Figure 2: The Majority of Life Cycle Costs are Locked by Concept Design ${ }^{1}$ completion of concept design the majority of project life cycle cost has been fixed (or rendered unchangeable) for a given design. Such extraordinary leverage presents a business case for conducting concept design in a rigorous and pragmatic fashion, particularly for immature mission concepts that advance the state of the art and that have high design uncertainty.

Done well, concept design can provide an executable system level design baseline for project teams in Phase B and later phases. Done poorly, it can lead to several undesirable outcomes for project teams, such as: a) cost overruns, b) the need for redesign (or for multiple redesigns characterized by fluid technical baselines with ever-decreasing capabilities), c) recurring schedule delays, d) contract disputes or cancellations, and e) challenges in retaining trained personnel.

Pre-Phase A and Phase A Offer Unique Venue for System Level Trades. As the design progresses from pre-Phase $\mathrm{A}$ into Phase $\mathrm{C}$, the scope of design work ranges from broad and shallow to narrow and deep. In Pre-Phase A and Phase A, concept design teams conduct broad and shallow trade studies to quickly evaluate a range of mission "requirements" 2 and design characteristics (R\&DC) and make key decisions that determine the final concept design. The concept design phases provide an ideal, inexpensive venue for conducting these system level trade studies, as teams in these phases typically are small, closely coordinated, and agile in their ability to handle high rates of change in system level R\&DC. Additionally, concept design teams typically operate absent many of the formalities present in later project phases. For example, typically there are no prime contracts, and system level requirements typically are not placed under formal configuration control until late in Phase A.

Conversely, Phase B and later phases are not well suited for system level trades. In Phase B, the system level design is more difficult and more expensive to change. Typically teams are larger and more distributed, prime contracts are in place, and system level requirements are under formal configuration control. Preliminary design work assumes the system level concept design is complete. In this more formal environment, collateral impacts (e.g., design, cost, contractual) of system level changes can become significant considerations, as can the time and effort required to conduct the configuration control process. For similar reasons, the system level design is even more difficult and more expensive to change during Phases C and D. In Phases C and D, teams typically are even larger, system and subsystem level requirements are under formal configuration control, and detailed design work either is underway or has been completed.

Illustrating these points, par. 2.1 of ref. (a) cites the historical data ${ }^{3}$ in Fig. 3 and states "NASA experience shows a positive correlation between a fully adequate mission design process (PrePhase A, Phase A, and Phase B) and a successful Phase C/D". Paragraph 2.1 of ref. (a) further states "It is generally accepted that Phase C/D cost overruns are usually created by a lack of understanding, inadequate definition of, and changing requirements." Reference (a) defines the

\footnotetext{
${ }^{1}$ Figure 2 is an excerpt from ref. (c) that has been reformatted for presentation in this paper.

${ }^{2}$ In this paper, the term "requirements" is used in an informal context when in quotes to denote interim reference capabilities used to guide evaluation of point designs within the trade space. System level requirements are not formally baselined until System Requirements Review (SRR) near the end of Phase A for a final concept design that meets technical and programmatic (including cost and schedule) constraints.

${ }^{3}$ From ref. (a), Fig. 2-1. A similar figure is published at ref. (d).
} 


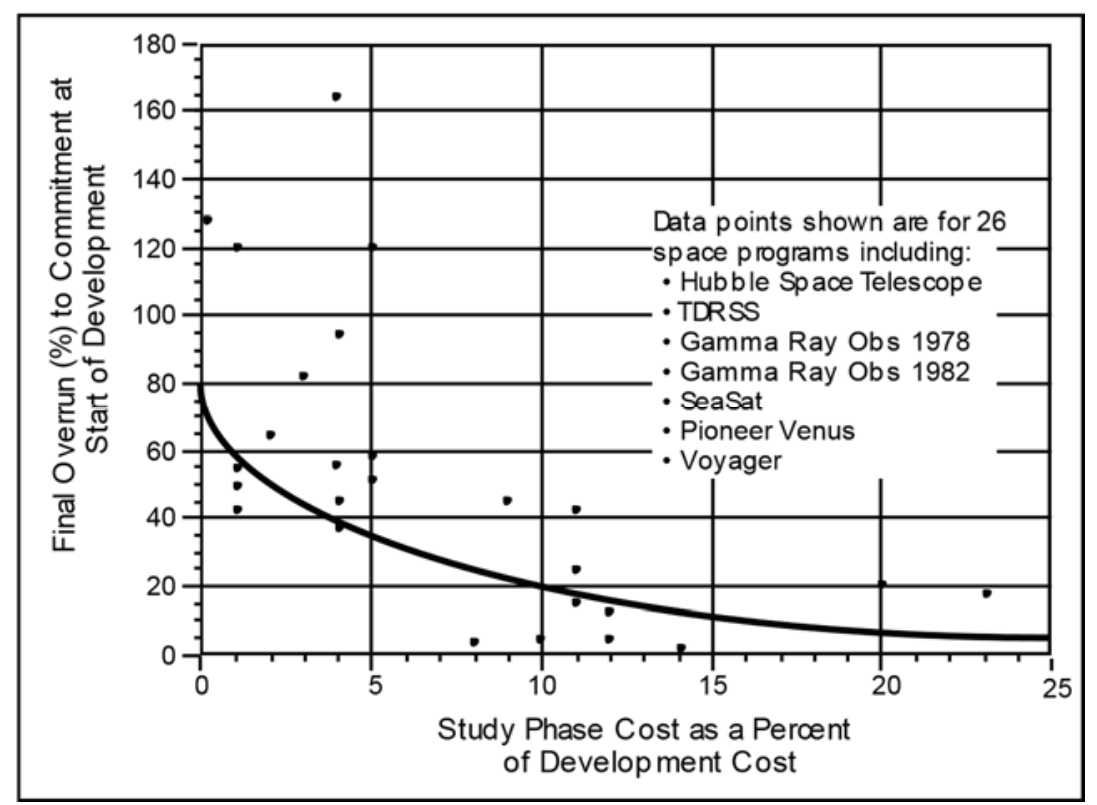

Figure 3: Benefit of Study Phase Investment (ref. (a), Fig. 2-1)

mission design process as occurring in the study phases, which ref. (a) further defines as prePhase A, Phase A, and Phase B. While the content of Fig. 3 is dated, and while the phase definitions in ref. (a) differ somewhat from those in Fig. 1 (i.e., in ref. (a), Phase B ends at SDR and does not include PDR), the general findings of ref. (a) remain relevant.

\section{Fidelity Needed in Concept Design Solution}

A Proposed Definition for a "Feasible" Mission Concept. Reference (a), par. 5.3 discusses a "feasible" design as one that "falls within mission requirements or budgetary guidelines". But in practice, the use of the term, "feasible", often is problematic. It is frequently used without definition, leaving open to interpretation whether a feasible design needs to be both technically feasible as well as cost and schedule constrained to a certain level of confidence, or whether it simply needs to be an early analytical proof of concept. The broad range of potential interpretations has significant implications on design teams, customers, and stakeholders.

This paper defines a "feasible" mission concept to mean:

Technical, cost, and schedule characteristics for a single, baseline mission concept design have been credibly converged to the first order by the end of Phase A, such that the design may be developed, launched, operated, and decommissioned by a competent project team starting in Phase B within customary technical and programmatic margins.

A Model for Product Fidelity in Design Phases. When conducting concept design, teams, customers, and stakeholders may seek an objective measure for required product fidelity to help them understand when a concept design study may be considered complete (i.e., to understand "how good is good enough?"). Such an objective measure would help avoid determining completion simply based on external considerations, such as when time or study funds have been exhausted, or such as pressure to meet a milestone on a high visibility, politically important effort. But measures for product fidelity tend to be general or qualitative in nature. 
For example, paragraph 4.4.1.2 of ref. (e) notes design teams conduct design solution analysis in a recursive and iterative fashion to successively increasing levels of refinement and resolution. It states "the design effort [proceeds] to a depth that is sufficient to meet several needs: the design must penetrate sufficiently to allow analytical validation of the design requirements; it must also have sufficient depth to support cost modeling and to convince a review team of a feasible design with performance, cost, and risk margins." Figure 4.4-3 of ref. (e) addresses levels of error or uncertainty, but only in a general sense.

A Proposed Metric for Level of Convergence. In the following paragraphs, a level of quantification is introduced for product fidelity that suggests the needed fidelity in product system level sizing and performance (SLSP) for the design phases is defined as having credibly achieved: a) first order convergence at the end of Phase A, b) second order convergence at the end of Phase $\mathrm{B}$, and c) third order convergence at the end of Phase C. Examples of SLSP parameters include system level mass and power.

This needed convergence is illustrated by the solid black, uniform convergence curves in Fig. 4 (adapted from ref. (a), Fig. 3-4), where allowable SLSP error decreases as the design progresses from Phase A through Phase C. Credible convergence to the first order by the end of Phase A

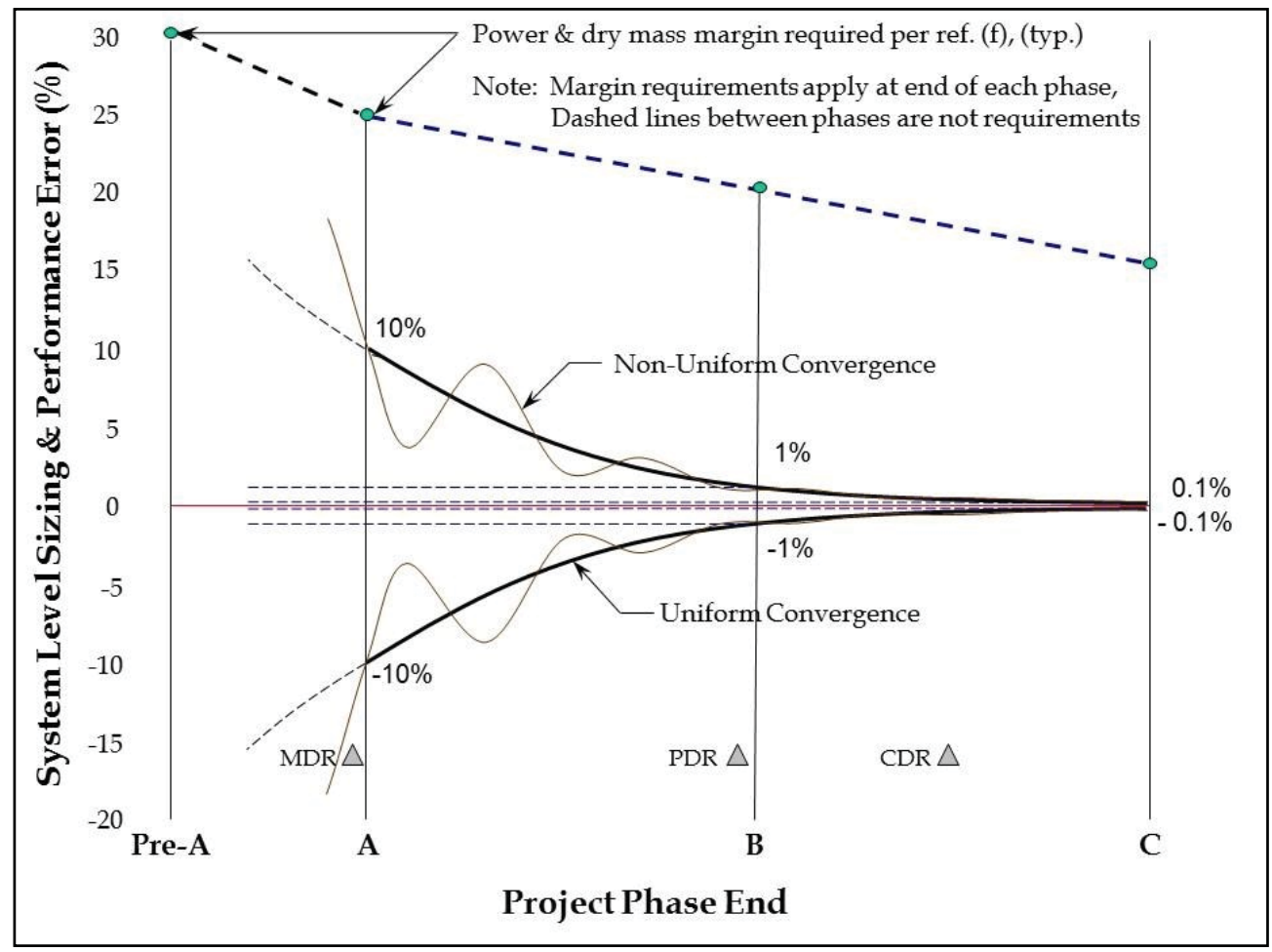

Figure 4: A Proposed Model for Product Fidelity in Design Phases

means SLSP of the mission elements has been confidently determined to within $90 \%$ (read this as accurate to one significant digit, or as $9 \times 10^{1} \%$ ) of what it will be when the flight system is delivered, for given cost and schedule constraints. This implies, for example, there is residual uncertainty that the design could get larger or smaller by $\sim 10 \%$, or that performance could increase or decrease by $\sim 10 \%$, between the end of Phase A and launch. The solid black curves also depict by the end of Phases B and C that SLSP has been confidently determined to the second order, or 
$99 \%$ (accurate to two significant digits, approximately $\pm 1 \%$ error), and to the third order, or $99.9 \%$ (accurate to three significant digits, approximately $\pm 0.1 \%$ error), respectively.

The metrics for SLSP error in Fig. 4 are intended as approximate guidelines only. They provide a coarse model that depicts an idealized trend of fidelity in each phase. Actual convergence may appear non-uniform, as also notionally depicted in Fig. 4. An SLSP error in this context typically means while sizing or performance calculations were done properly, they were done with incomplete or incorrect information and/or assumptions. Implicit in this model is SLSP values at the end of Phase C are within the range of possible SLSP values at the end of Phase A. Also implicit is SLSP values include contingency quantities typically added to address the uncertainty of immature items, such as items that have a low technology readiness level.

Example SLSP Error Convergence. To visualize an example of SLSP error for mass, consider that by the end of Phase A, mass for a 4,000 kg observatory should be known to within approximately $\pm 10 \%$, or $\pm 400 \mathrm{~kg}$, of its final launch mass. As the design matures in subsequent phases, concept phase estimates are replaced by more refined estimates, or by actual flight hardware masses. By the end of Phases B and C, mass for a 4,000 kg observatory should be known to within approximately $\pm 1 \%$ and $\pm 0.1 \%$ (or to within approximately $\pm 40 \mathrm{~kg}$ and $\pm 4 \mathrm{~kg}$ ), respectively, of the final launch mass.

Role of Resource Margins on Needed Convergence. The needed SLSP error convergence in Fig. 4 must be within the envelope of the required design margins. Figure 4 shows examples of design margin requirements selected from ref. (f), where power and dry mass margins on flight systems are required to be $\geq 25 \%, \geq 20 \%$, and $\geq 15 \%$ at the end of Phases A, B, and C, respectively. Cost and schedule margin requirements apply from ref. (g) as well, though not depicted in Fig. 4. For example, ref. (g) requires projects at the start of Phase $\mathrm{C}$ to have a budget margin of $\geq 25 \%$ through Phase D. It also levies as a goal (not a requirement) that projects at the start of Phase B have a budget margin of $\geq 30 \%$ through Phase D.

Importance of Concept Design Convergence to Project Managers. Project managers and project teams conducting product design and development in Phases B-D rely on having received a concept design that has been credibly converged to the first order by the end of Phase A, so they can execute their projects within customary allocations for technical, cost, and schedule margins. ${ }^{4}$ To illustrate the effect margins have on required convergence, consider a project manager (PM) who receives a completed mission concept design at the start of Phase B. This PM can accommodate a concept design that has been credibly converged to within $10 \%$ of its flight sizing and performance values for power and dry mass resources without redesign, even if the $10 \%$ error occurs in the direction of needing increased resources, since the mission elements (including the launch service) already have been sized to accommodate a $25 \%$ growth margin. Conversely, this PM cannot accommodate a concept design that has been credibly converged only to within $30 \%$ of its flight sizing and performance values for power and dry mass, if the $30 \%$ error occurs in the direction of needing increased resources. The design will likely need to be de-scoped in Phase B or later phases.

\footnotetext{
${ }^{4}$ Margins typically are used to accommodate unforeseen needs or events.
} 


\section{Techniques for Designing the Mission Level Trade Space}

A design-to-cost trade space such as shown in Fig. 5 can be developed to expedite convergence of the concept design. This trade space has axes of mission life cycle cost (referred to here also as just "cost"), technical capability, and development schedule. ${ }^{5}$ Absent other guidance, each of these axes has equal importance, i.e., each can be viewed as one leg of a three-legged stool.

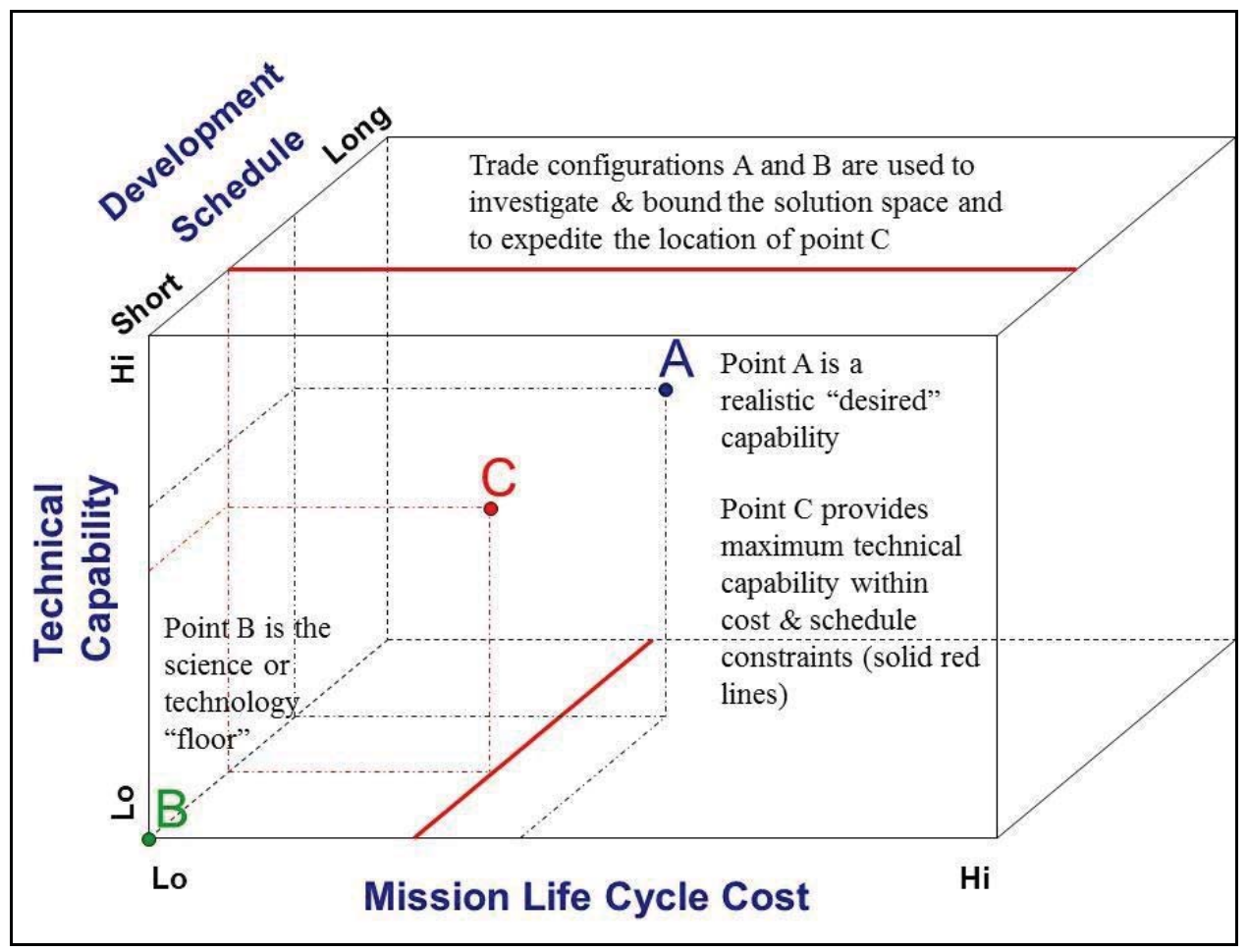

Figure 5: 3-Cycle Mission Level Concept Design-To-Cost Trade Space

Figure 5 shows a trade study structured to converge in three discrete design "cycles". Within the trade space, there are two bounding design points, labeled "A" and "B". The A design reflects a realistic "desired" capability. The B design reflects a science or technology "floor" capability, i.e., the point below which the mission is no longer compelling from a science or technology perspective. An intermediate design point (labeled "C") maximizes technical capability (i.e., science or technology return) for the cost and development schedule constraints depicted by the solid red lines. ${ }^{6}$ The technical capability of the $\mathrm{C}$ design is not known at the outset of the study. The goal is to credibly determine point $\mathrm{C}$ with minimum expended time and effort.

The approach in Fig. 5 deduces R\&DC for the $\mathrm{C}$ design by interpolating on the results from the $\mathrm{A}$ and $\mathrm{B}$ designs. It is more like a root finding algorithm than like the spiral (e.g., successive refinement ${ }^{7}$ ) design processes typically used in Phases B and C. In Phases B and C, each design typically is a refinement of the baseline system level design from the prior phase. In the concept design process described here, typically there is no baseline system design until the concept design

\footnotetext{
${ }^{5}$ Figure 5 provides a convenient visualization for depicting design results in the context of key constraints. As technical capability is increased, cost and/or schedule typically increase. Alternatively, for a given technical capability, a schedule increase may result in a cost increase, etc.

${ }^{6}$ Cost and schedule constraints often are given in request for proposal solicitations. Other constraints may apply as well, such as technology readiness, risk, etc. ${ }^{7}$ Ref. (e), Fig. 4.4-2
} 
is complete. While some aspects of the $\mathrm{A}$ and $\mathrm{B}$ designs may endure in the $\mathrm{C}$ design, neither the A design nor the B design is intended to represent the final concept design. Instead, the systematic process described here is designed to purposely guide the team to view the design problem from multiple perspectives. This helps illuminate unexpected findings (e.g., accelerates the discovery of "unknown unknowns") that otherwise may have remained hidden from view. It also quickly educates the team on the implications of the bounding "requirements", stimulates creative thinking, and helps mitigate biases while reducing design uncertainty.

For this discussion, the A design is assumed to be evaluated first. The order of evaluation for the first two design points is subject to team preference. There are positive and negative aspects to conducting either design first. For example, when the first design is the A design depicted in Fig. 5 , it is also the most complex design. This means the team will be evaluating the most challenging design while it is on the steepest part of the learning curve with respect to both the design and the study team operation. However, such an A design also may be the most welldefined design point, as often it will reflect either the customer's desired capability or a substantial fraction of that capability. Conversely, when the first design is the B design in Fig. 5, the design will be less complex. But it also may not be as well defined. Further, the science or technology floor often is easier to identify after conducting a design cycle and completing a full cost estimate. Without the benefit of a prior cycle to advise on mission cost, the team may not fully recognize the need to identify the R\&DC for a "true" science or technology floor.

Selecting R\&DC Effectively. Selecting bounding cases is key to this approach, as it allows the design team to interpolate within the bounds of its interim study results to select the R\&DC for the $\mathrm{C}$ design. Conversely, failure to select design cycle cases that prove to be bounding can cause the team to extrapolate beyond the bounds of its interim study results to determine the R\&DC for the $\mathrm{C}$ design. Extrapolation adds risk in the mission technical, cost, and development schedule estimates. The need to extrapolate could occur, for example, if the team selects R\&DC for the A and $\mathrm{B}$ designs that result in each design exceeding cost and schedule constraints. This would indicate the team did not take a big enough step down to identify the true science or technology floor for the B design (this presumes a solution exists). Alternatively, not reaching the true science floor on the B design may incur the need to perform additional design cycles in order to credibly determine the R\&DC for a final solution that meets cost and schedule constraints. This may be a critical failure if the final design is not available by the required deadline, or it may incur team overtime. In some instances, teams may find, after exhausting all reasonable options, there is no acceptable solution that meets cost and schedule constraints. This is a valid finding that is far less costly to learn in concept design than later in the life cycle.

Optimistic A designs and "false" science floors for B designs are common when establishing the R\&DC for the A and B designs. The customer (e.g., a scientist / principal investigator) often provides a major input into the R\&DC for the A and B designs. However, the customer's vision for the A design often is not cost and schedule constrained, as it has not yet been informed by rigorous cost and schedule analysis. Further, the customer may resist identifying the true science or technology floor for the B design out of concern it may become baselined as the final concept design. Teams that recognize, or adapt to, these considerations pragmatically and quickly typically will fare better than teams that do not. 
Selecting truly bounding R\&DC cases for the A and B designs objectively and without bias will help the design team make the best use of its limited design cycles. In a typical case, most (but not necessarily all ${ }^{7} \mathrm{R} \& \mathrm{DC}$ parameters: a) reflect a relatively high, but realistic technical capability with a relatively long development schedule for the A design, b) reflect a science or technology floor with a relatively short development schedule for the B design, and c) are between the A and $\mathrm{B}$ design $\mathrm{R} \& \mathrm{DC}$ for the $\mathrm{C}$ design. The $\mathrm{R} \& \mathrm{DC}$ set for the $\mathrm{B}$ design is reevaluated after the $\mathrm{A}$ design cycle to assure the solution space remains bounded.

Many parameters vary between the A, B, and C designs, a byproduct of having to conduct a fully coupled $^{9}$ investigation over a broad solution space in limited time. ${ }^{8}$ With such an approach, it may appear so many parameters vary between the $\mathrm{A}, \mathrm{B}$, and $\mathrm{C}$ designs that it will be prohibitively difficult to understand the sensitivity of a specific design characteristic to a specific design "requirement". However, experience with this approach on multiple studies at the NASA Goddard Space Flight Center has shown teams have been able to gain sufficient understanding of parameter sensitivities over the course of a design study.

\section{Challenges in Determining Credible Design Convergence}

For a design to be considered credibly converged at the end of Phase A, convergence variations need to be damped such that SLSP error is sustainably $\leq 10 \%$. One of the most vexing aspects of concept design is specifying an objective criterion that indicates the design has credibly converged to the first order, because convergence often is evident only in hindsight. This occurs because concept design teams learn at a high rate and because design and cost uncertainty is high. As teams progress through the design cycles, some of that learning alters the technical, cost, or schedule results of prior cycles.

A subjective criterion is concept design teams normally experience at least a few significant surprises, including discovery of significant unknown unknowns, during the course of a study. Teams that have not experienced significant surprises should be cautious of their results and should recognize the residual risk of discovering significant surprises in later development phases. A lack of significant surprises may indicate a team has not sufficiently: a) progressed down the learning curve, b) exercised the trade space, or c) mitigated biases. It also may indicate the design did not represent a significant advance in the state of the art. Not all surprises will appear in the design. For example, utility analyses conducted by science team members may unexpectedly reveal new utility in science observations which may alter the science value models in a way that better accommodates design constraints or otherwise facilitates the design.

Why Early Cost Estimates Tend to be Optimistic. A common characteristic of concept design is costs for a given design tend to increase with each design cycle. This typically occurs because as teams progress through future design cycles, they learn successively more about what may have been omitted in previous cycles. Figure 6 notionally illustrates this, where after the B design cycle is complete, the cost of the A design increases for a given technical capability.

\footnotetext{
${ }^{7}$ e.g., R\&DC for the B and C cycles may contain selected R\&DC parameters from prior cycles, as appropriate ${ }^{9}$ The use of "fully coupled" means the effects of a "requirement" or a design attribute are assessed concurrently across all subsystems for all elements (e.g., spacecraft, instrument, etc.).

${ }^{8}$ After an approach used by Mr. John Oberright, NASA / GSFC Emeritus, for the Space Technology-5 concept design study (1999)
} 


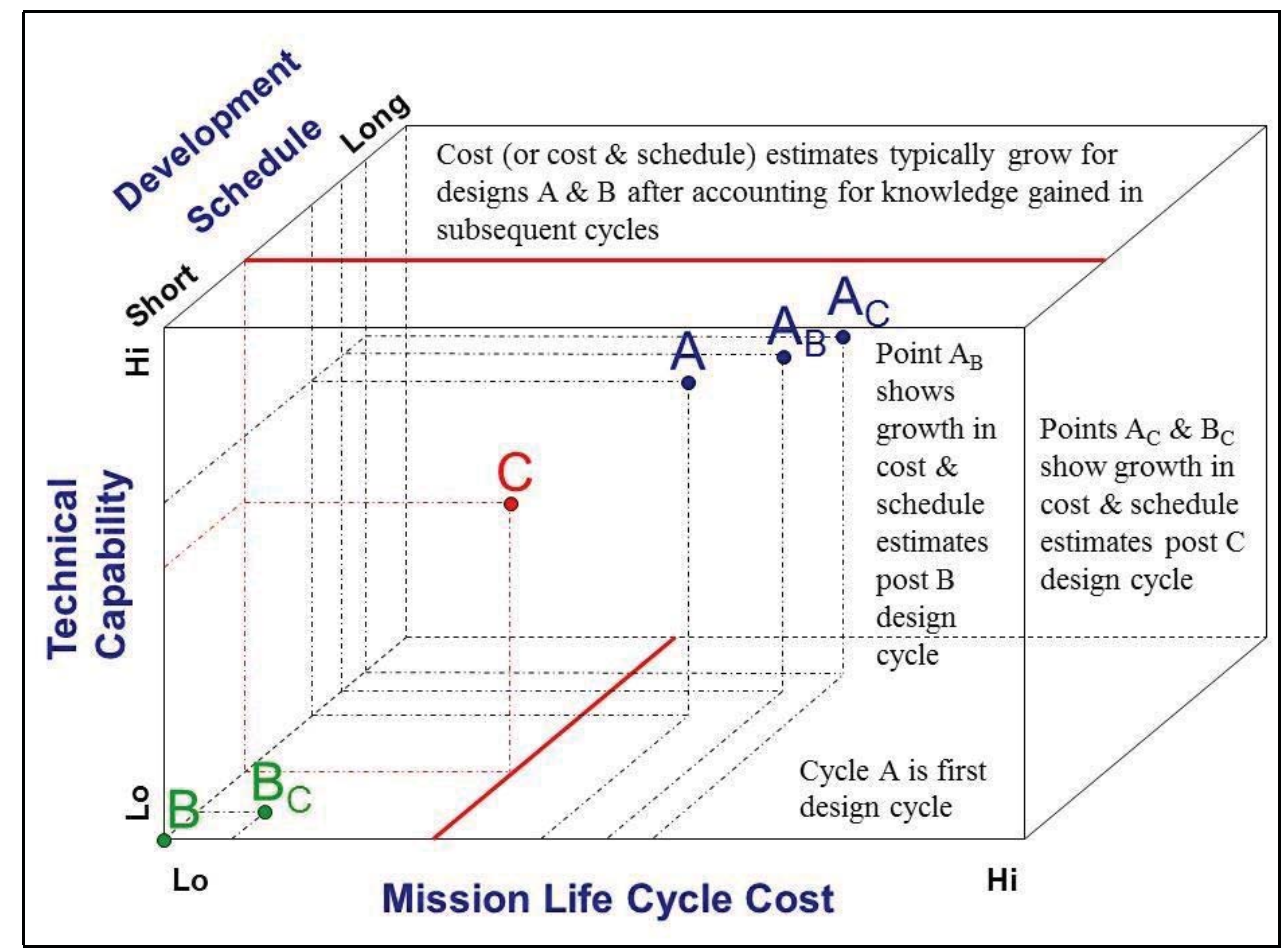

Figure 6: Convergence Determinations Often are Evident Only in Hindsight

Similarly, after the $\mathrm{C}$ design cycle is complete, the cost of the A design increases again, and the cost of the B design increases for a given technical capability. Apparent only in hindsight, teams should be aware this effect is characteristic of most design studies for immature mission concepts that advance the state of the art and that have high design uncertainty. Following the $\mathrm{C}$ design cycle, this learning tends to taper off for most designs if the A and B designs were bounding cases. When schedule increases accompany these cost increases as in Fig. 6, the A and B design points move not only to the right, but also into the page.

Both Design and Work Breakdown Structure (WBS) are Key to Cost Fidelity. Concept design teams typically perform cost analysis using multiple methods. One of these methods is "grass roots", which uses a WBS. In this method, each WBS element has a corresponding WBS dictionary definition. These definitions, along with team costing ground rules, help teams account for costs consistently among mission elements. The WBS dictionary for most space mission elements, e.g., spacecraft, launch, ground systems, etc., is largely existing and relatively well known. Conversely, the WBS dictionary for a new instrument is unique. It evolves as the instrument design evolves. This can be a key factor in estimating costs when the mission concept is dominated by a new instrument, as most of the mission development costs may be associated with the least well understood mission element. Teams typically need multiple design cycles not only to develop a well understood design, but also to develop well understood cost estimates that are free of significant gaps and overlaps and that apply team costing ground rules consistently.

Four Cycle Process. While Figs. 5 and 6 show a three design cycle process, a four cycle process may be used when time and resources permit. Using four cycles allows more refined coverage of the solution space prior to entering the final ("D") design cycle. That is, completing three points prior to the final design offers the opportunity to identify a "knee in the curve", whereas having two points provides only linear trends. A four cycle process also allows more opportunities to vary 
subsystem implementation choices and reduces the number of parameters that must be varied concurrently in each cycle.

\section{Additional Recommended Practices for Conducting Mission Concept Design Studies}

Treat Design Cycles as a Precious Resource. Design cycles are essential for determining a concept design solution, but they are also in limited supply due to the time and manpower available. Team efforts should focus on what is needed to directly develop the product and should actively limit peripheral tasks and activities. Teams typically should not attempt to retrofit the A and B designs with insights gained from the $\mathrm{B}$ and $\mathrm{C}$ designs, respectively. Time usually is better spent just applying learning from the $\mathrm{A}$ and $\mathrm{B}$ designs to the $\mathrm{B}$ and $\mathrm{C}$ designs, respectively. Maintaining a list of these insights for the A and B designs, however, is often useful.

First Cost Estimate Should Not be Final Cost Estimate. A team should not let its first cost estimate be its final cost estimate. Due to unknown unknowns and learning curve effects, early design and cost results may not be as they initially appear.

Maintain First Order Analysis in Concept Design. Pre-Phase A and Phase A teams evaluate multiple designs across a broad trade space in a relatively short period to credibly localize the final concept design. Analysis tools typically used are representative to first order precision and agile enough to adapt to frequent and significant changes at the system level. ${ }^{9}$ By comparison, analysis tools typically used in preliminary design (Phase B) are representative to second order precision and assume the system level design is stable. Similarly, analysis tools typically used in detailed (or final) design (Phase C) are representative to third order precision and assume both the system and subsystem level designs are stable.

A coarse, but useful, analogy is to consider work performed in: a) pre-Phase A and Phase A as being done with a hacksaw, b) Phase $\mathrm{B}$ as being done with a file, and c) Phase $\mathrm{C}$ as being done with a polisher. When a team is found using a hacksaw in Phase $\mathrm{C}$, it has done something wrong. Such a team did not credibly converge the first order solution by the end of Phase A such that it would remain stable in later phases. As a result, the team is having to redo system level concept design work late and out of sequence. Similarly, a team found using a polisher in Phase A is doing something wrong. Such a team will not move quickly or broadly enough to rough-out and credibly converge a first order solution in the time available. Reinforcing this point is some elements of early concept designs may not even exist in the final concept design. Polishing these elements can result in valuable time lost.

Avoid Significant Rounding Errors. To avoid masking resource margins, teams typically should bookkeep internal design and performance calculations to three significant digits and report results to two significant digits. ${ }^{10}$ Note that this should not be taken to imply there is three-digit accuracy in concept design work. There usually is not. This practice is simply a numerical safeguard to

\footnotetext{
${ }^{9}$ In some cases, such as new or mission critical technology areas, more in-depth analysis may be selectively warranted to reduce cost, technical, or schedule risk.

${ }^{10}$ This is a minimum guideline.
} 
avoid propagating rounding errors into the second significant digit where these errors potentially could overwhelm the ability to adequately determine design or performance margins.

Computing meaningful design and performance margins in concept design study products depends, in part, on having sufficient fidelity in the numerical values used to compute those margins. Insufficient fidelity due to rounding errors can overwhelm margin calculations when teams do not use sufficient numerical safeguards. For example, consider the two cases below where power margin is calculated per ref. (f).

Case 1: $\quad$ Power Available

Max. Estimated Power Required

$=200 \mathrm{~W}$

Power Margin = $100(200 \mathrm{~W}-249 \mathrm{~W}) / 249 \mathrm{~W}$

$=249 \mathrm{~W}$

$=-19.7 \%$

Case 2: $\quad$ Power Available

$=200 \mathrm{~W}$

Max. Estimated Power Required

Power Margin = $100(200 \mathrm{~W}-151 \mathrm{~W}) / 151 \mathrm{~W}$

$=151 \mathrm{~W}$

$=32.5 \%$

The margins for Cases 1 and 2 are $-19.7 \%$ and $+32.5 \%$, respectively. Now consider a third case in which a designer rounds calculations to the first digit in Cases 1 and 2.

Case 3: $\quad$ Power Available

Max. Estimated Power Required

$=2 \times 10^{2} \mathrm{~W}$

Power Margin $=100\left(2 \times 10^{2} \mathrm{~W}-2 \times 10^{2} \mathrm{~W}\right) / 2 \times 10^{2} \mathrm{~W}=0 \%$

The calculation for Case 3 shows zero margin and illustrates how rounding can have a significant effect on margin determination. From Fig. 4, the power margin at the end of pre-Phase A is required to be $30 \%$. Comparing Case 3 with Case 2 shows this required $30 \%$ margin can be fully masked when rounding to the first digit. Additional rounding errors can accrue when combinations of rounded results are used to perform successive calculations.

Document Study Results in Reports. Per ref. (h), formal trade studies should be well documented, i.e., "formal trade studies ... are typically well-documented and become part of the decision database normal to systems development". This guidance applies to concept design results, as concept design consists principally of formal trade studies.

Design and trade study results should be documented in reports for each subsystem and discipline at end of each cycle. These reports provide the official study record of what team did, how the team did it, and what the team found for present (and future) team use. Built from standardized templates, their technical descriptions include analysis methods and example calculations. These reports enable effective: a) technical integration across subsystems and disciplines, b) system level review and integration, and c) independent review. They also provide coherent and comprehensive technical waypoints that enable team members to recall design and performance information from prior cycles that often is needed for design scaling or comparison. Absent such waypoints, the high rate of design changes in concept design makes recalling such information difficult. These technically functional (but not necessarily editorially pristine) reports typically are approved by, and maintained under informal configuration control of, the systems study engineer. Briefings 
often are too cursory to effectively serve these purposes. Briefings, if needed, may be quickly built from the approved reports and contain only information contained in the approved reports.

Recognize Four Unofficial, but Typical, Phases of Concept Design. Concept design teams developing immature mission concepts that advance state of the art often experience four sequential phases of work. Recognizing and understanding these phases is key to guiding a team to determine a feasible concept design within the allotted study time and resources.

1) Unbridled Optimism: This phase features unbridled, optimistic performance desires levied as "requirements" before a team gains a credible understanding of the associated cost and schedule implications. Meetings often are not well-focused on study objectives. Instead, they feature extended advocacy discussions (e.g., why the mission has the best science of all competing missions, why the mission has the best chance to win, etc.).

2) Shock: This brief phase usually begins after a team completes its first credible cost estimate.

3) Denial: This phase features abundant rationalizations as to why the models used for the cost estimate were not representative. A team points to any aspect of the mission - except the excessively high technical capability - as the reason costs are too high, so that science return remains compelling relative to that of the competition.

4) Acceptance: This phase features the ultimate realization that technical capability / science return must be lowered to design a credible mission concept that meets cost and schedule constraints according to established independent review standards.

Time spent in phases 1-3 usually is non-recoverable. Teams that dwell in these phases often experience significant overtime demands as they approach study completion on a fixed study timeline. The more quickly a team moves through Phases 1, 2 and 3, and arrives at Phase 4, the better that team is likely to fare.

\section{Closing Thoughts}

Given the extraordinary leverage concept design has in determining life cycle cost, there is a business case for conducting concept design in a rigorous and pragmatic fashion, particularly for immature mission concepts that advance state of the art and that have high design uncertainty. This paper provides specific techniques in trade space design and trade study execution to help achieve credible design-to-cost convergence with minimum expended time and effort. It introduces a level of quantification for the degree of convergence needed in the concept design product in the context of technical and programmatic resource margins available in preliminary design. It also identifies challenges that can mask credible convergence, including design and cost evaluation learning curves, unknown unknowns, and numerical errors. In addition, it provides techniques for selecting trade study cases that help manage those challenges and that promote objective concept evaluation, help reveal unknowns, and expedite convergence.

The concept design phases provide a unique venue to facilitate exploring and converging the system level design. Done well, concept design can provide an executable system level design baseline for project teams in Phase B and later phases. When not done well, some of the work of 
the concept design phases usually will have to be done again. The later this realization occurs, the more expensive the resulting redesign is likely to be.

\section{References}

a) Ryschkewitsch, Michael G., ed. 1992. The NASA Mission Design Process, An Engineering Guide to the Conceptual Design, Mission Analysis and Definition Phases. Washington, DC (US): NASA Engineering Management Council, NASA Headquarters. 22 December.

b) NASA Headquarters. 2012. NASA Procedural Requirements (NPR) 7120.5E w/Changes 1-10. NASA Space Flight Program and Project Management Requirements, Figure 2-5. Washington, DC (US): Office of the Chief Engineer.

c) Strategy Bridge International, Inc. 2013. "Fundamentals of Systems Engineering". 5th Ed. Presented at NASA Academy of Program/Project \& Engineering Leadership training. Day 3, Chart 44. NASA/Goddard Space Flight Center, Greenbelt, MD (US). 11-15 February.

d) NASA Headquarters. 1995. NASA/SP-610[5]. NASA Systems Engineering Handbook. Figure 6. Washington, DC (US): Office of the Chief Engineer.

e) NASA Headquarters. 2007. NASA/SP-2007-6105 Rev 1. NASA Systems Engineering Handbook. Washington, DC (US): Office of the Chief Engineer.

f) NASA/Goddard Space Flight Center. 2013. GSFC-STD-1000F with Administrative Changes.

Goddard Space Flight Center Rules for the Design, Development, Verification and Operation of Flight Systems. Table 1.06-1. Greenbelt, MD (US).

g) NASA/Goddard Space Flight Center. 2008. Goddard Procedural Requirements (GPR) 7120.7 w/Administrative Extension 2. Schedule Margins and Budget Reserves to be Used In Planning Flight Projects and In Tracking Their Performance. Greenbelt, MD (US).

h) Defense Acquisition University. 2001. Systems Engineering Fundamentals. Paragraph 12.1. Washington, DC (US): Office of the Undersecretary of Defense for Acquisition, Technology, and Logistics.

\section{Biography}

Mr. Di Pietro is the Senior Engineer for Advanced Concepts and Architectures at the NASA Goddard Space Flight Center where he has led, or served as mission systems engineer on, over 20 advanced space mission designs. He has served in interagency assignments with the Defense Department in space architecture development and with the State Department in defense trade controls. His prior assignments include Delta II Launch Service Manager (NASA), Space Station Freedom Thermal Control System Manager (NASA), and aircraft advanced design engineer (Naval Air Systems Command). He has M.S. and B.S. degrees in Aerospace Engineering and is a retired Colonel, US Air Force Reserve. 